\title{
Immediate post-abortal insertion of the frameless GyneFix IUD: initial experience in China
}

\author{
Xiaoming Cao, MD \\ Director \\ Wenhao Zhang, MD \\ Co-worker
}

Yajie Gao, MD

Co-worker

Xan Zhao, MD

Co-worker

Nong Lin, MD

Co-worker

Limei Wang, MD

Co-worker

Chanhna Li, MD

Co-worker

Lijnan Song, MD

Co-worker

Weijia Zhang, MD

Co-worker

\section{Zhihong Zhang, MD \\ Co-worker}

Tianjin Municipal Institute for Family Planning, Tianjin, China

Henry Van Kets, MD, PhD

Professor, Department of Obstetrics and Gynaecology, University Hospital, Ghent, Belgium

Dirk Wildemeersch, MD

Director, Contrel Research,Piers de Raveschootlaan 125, 8300 Knokke, Belgium

(Accepted February $\left.2^{\text {nd }}, 2000\right)$

\section{Summary}

The promising results obtained with the frameless GyneFix IUD for interval insertion ${ }^{l}$ led to the belief that the technology could be useful for immediate post-abortal application to reduce the number of induced and repeat abortions. IUDs have many advantages and the method is acceptable and safe. However, side effects and expulsion of conventional IUDs remain a problem. In an attempt to minimise these problems, the frameless GyneFix IUD was developed. Clinical studies conducted over the past 14 years have shown that the principles on which the device is based are valid. An increasing bulk of clinical evidence shows that the design characteristics of the device (fixed, frameless and flexible) are responsible for the low expulsion, high effectiveness, and high tolerance and continuation rates. ${ }^{1}$ Experience with the frameless IUD in China since 1989 confirms the international experience with the device. ${ }^{2,3}$

The present communication is a second report from China with the GyneFix IUD. In the present study the GyneFix IUD is inserted immediately following termination of pregnancy of less than 10 weeks amenorrhea. It is
\end{abstract}

concluded that immediate post-abortal insertion of the frameless IUD is easy and safe, and appears to be as reliable and as effective as when it is inserted at interval. The GyneFix IUD could, therefore, constitute an important new option in the prevention of abortion.

Key words

frameless intra-uterine device, GyneFix, post-abortal insertion

\section{Key message points}

- The number of unintended pregnancies and induced abortions continues to rise in developed and developing countries.

- IUDs are extremely useful as they can be inserted immediately following induced abortion in women without pelvic infection or septic abortion.

- Partial or complete expulsion of conventional IUDs is a problem that may lead to early discontinuation.

- The immediate post-abortal insertion of the frameless anchored IUD results in low expulsion, high effectiveness and high continuation rates.

- Skilful insertion of the frameless IUD may be an important new and affordable contraceptive option to prevent repeat abortion. 


\section{Introduction}

In China, $40 \%$ of fertile women rely on IUDs for birth control. ${ }^{4}$ Sterilisation is used in China by more than $50 \%$ of couples $(40 \%$ female and $11 \%$ male sterilisation, respectively $\left.{ }^{4}\right)$. All other methods are seldom used. The Pill and injectables are used by only $3.6 \%$ of women. ${ }^{4}$ Birth control methods which rely on the daily administration of contraceptives (pills) are less suitable than long-acting methods to help reduce the number of accidental pregnancies and induced abortions. The use-effectiveness of the Pill is much lower than most service providers perceive. The typical user failure rate of the Pill per 100 users at 1 year is at least $5 \%$, and is related to incorrect and inconsistent use since the pregnancy rate in perfect users is much lower $(0.1 \%) .^{5}$

Methods which are not dependent on daily administration may be more efficient. For example, it has been shown that Norplant is much more effective than the Pill in preventing pregnancy in adolescent mothers. ${ }^{6}$ However, implants have certain drawbacks (e.g. bleeding disturbances) which are less acceptable to women.

Many women use abortion as a fertility-regulating method because modern contraceptive methods are not available, too expensive, or of poor quality., ${ }^{7,8}$ Most of the unplanned pregnancies end in abortion, particularly in young women. ${ }^{9}$ Induced abortion in adolescents and young women is rapidly increasing in many developed and developing countries, including China. ${ }^{10}$ Providing postabortal family planning services and methods suitable for young women is therefore extremely important. Although this should be the responsibility of the abortion provider, such services are often neglected. ${ }^{11,12}$

Ovulation returns almost immediately post-abortion: within 2 weeks for first trimester abortion and within 4 weeks for second trimester abortion. Within 6 weeks of abortion, $75 \%$ of women have ovulated. ${ }^{13}$ It is therefore of paramount importance that safe and effective methods of post-abortion contraception be provided to avoid repeat abortions.

Immediate post-abortal IUD insertion is a very convenient way to provide contraception at an opportune moment. At this time motivation for contraception is usually high, it is convenient for the woman as well as the provider, the cervix is dilated, and one has the assurance that the woman is protected before leaving the clinic. Additionally, the reduced cost may also be important. However, side effects and expulsion of conventional IUDs remain a problem. In an attempt to minimise these problems, the frameless GyneFix IUD was developed. Clinical studies conducted over the past 14 years have shown that the principles on which the device is based are valid. An increasing bulk of clinical evidence shows that the design characteristics of the frameless IUD (fixed, frameless and flexible) are responsible for the low expulsion, high effectiveness and high tolerance rates. ${ }^{1-3}$

The main objective of the study was to evaluate the retention of the GyneFix IUD when inserted immediately following surgical evacuation, by aspiration, of a pregnant uterus of less than 10 weeks amenorrhea. Important additional objectives were to evaluate the pregnancy and discontinuation rates for the GyneFix, and the safety of the anchoring technique.

\section{Methods}

No special training courses were considered necessary because the investigators already had some experience with the anchoring technique during interval insertion. This study was a single centre study, and it was approved by the local Ethics Committee of the Family Planning Institute.
Figure 1 GyneFix IUD inserted in the uterus

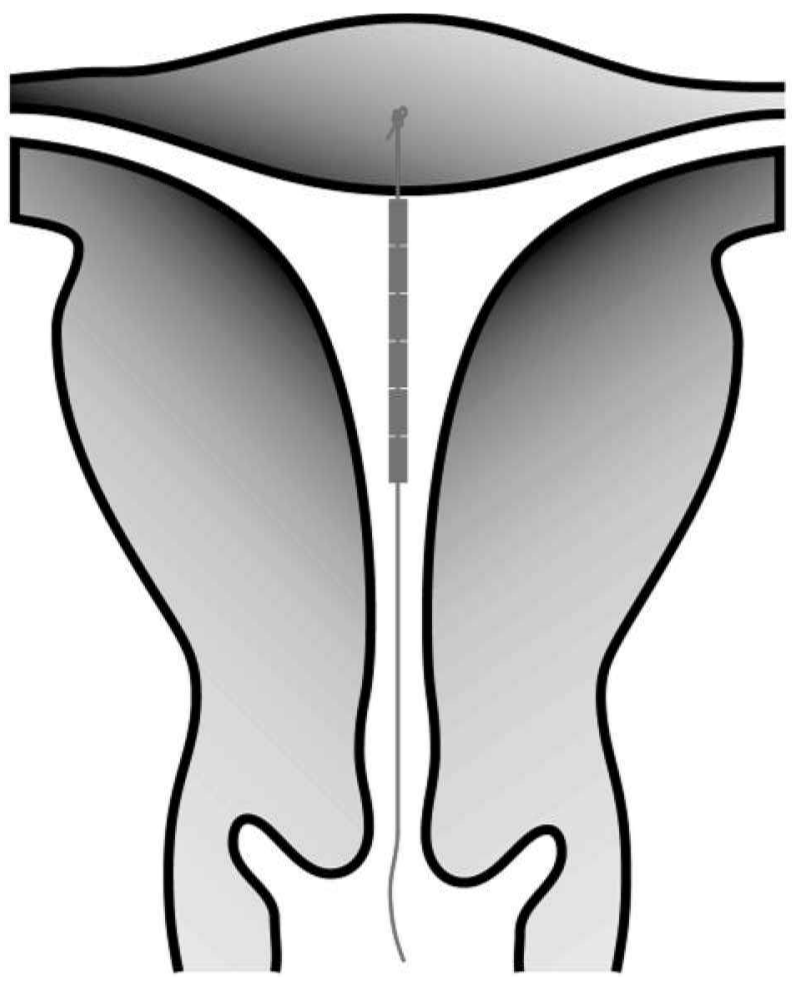

Description of the GyneFix IUD (Figure 1)

The GyneFix IUD and insertion technique have been described previously. ${ }^{1}$ The GyneFix lacks a plastic frame and this accounts for its flexibility. The anchoring system is essential for retention of the frameless device. Its minimum effective life is 5 years.

\section{Admission procedure}

All women volunteering for the study were screened for their clinical suitability for IUD insertion and compliance with the eligibility criteria. The following were excluded: nulliparous women, women with a history of pelvic inflammatory disease (PID) since their last pregnancy, pregnancy of more than 10 weeks amenorrhea, a history of ectopic pregnancy, recent sexually transmitted disease, undiagnosed genital tract bleeding, a congenital genital tract malformation, known or suspected genital malignancy, multiple uterine fibromyomas associated with menstrual disorders, clinical or laboratory evidence of anaemia as locally defined, or a history of trophoblastic disease in the last pregnancy. After giving consent for participation in the study, a medical, obstetric and gynaecological history was obtained from each subject.

A total of 175 post-abortal women were enrolled in the study from May 1995 to March 1999. The first insertion was done on $22^{\text {nd }}$ May 1995 and the last insertion on $30^{\text {th }}$ March 1999. Prior to insertion of the GyneFix IUD, proper contraction of the uterus was established. Oxytocin was given (10 IU intravenously or into the cervix) if the uterus was not well contracted.

\section{Follow-up procedures}

The subjects were requested to return for routine follow-up after the first menstruation, at 3,6, and 12 months after insertion, and yearly thereafter. Women were instructed to return to the clinic at any time if they experienced any problems with the device, and were free to return to the clinic at any time and request removal of the device. 
Data collection, monitoring and analysis

Data were recorded on standard pre-coded forms in duplicate at admission, at each scheduled and unscheduled follow-up visit, and upon discontinuation from the study. All data were sent to the data co-ordinating center at the Tianjin Institute for Family Planning where they were managed according to standard procedures. The data were analysed using the SAS statistical software package and the cumulative discontinuation rates were computed using the daily life table method. ${ }^{14-16}$

\section{Results}

\section{Subject characteristics}

The age and gravidity/parity distributions are given in Tables 1 and 2 . In 10 women data about the parity status are lacking. It is noteworthy that the previous pregnancy of almost all women was terminated. Fifty-five women indicated that they used some form of contraception before the current pregnancy: one used oral contraception; 25 used condoms; four used other non-defined methods and 25 used an IUD but either experienced expulsion or the device was removed.

Table 1 Characteristics of IUD users: Age distribution

\begin{tabular}{lcc}
\hline & No & $\%$ \\
\hline Average Age & 31.72 & \\
SD & 4.82 & \\
No & 175 & \\
Minimum & 21 & \\
Maximum & 43 & \\
Median & 31 & $(6.3)$ \\
$21-25$ & 11 & $(37.1)$ \\
$26-30$ & 65 & $(26.9)$ \\
$31-35$ & 47 & $(26.9)$ \\
$36-40$ & 47 & $(2.9)$ \\
$41-45$ & 5 & $(0.0)$ \\
$>45$ & 0 & \\
\hline
\end{tabular}

Table 2 Characteristics of IUD users: Gravidity and parity distribution (data on only 165 women)

\begin{tabular}{|c|c|c|c|c|}
\hline & Gravidity & $\%$ & Parity & $\%$ \\
\hline Average & 2.442 & 0.945 & & \\
\hline $\mathrm{SD}$ & 0.959 & 0.276 & & \\
\hline No & 165 & 165 & & \\
\hline Minimum & 1 & & 0 & \\
\hline Maximum & 7 & & 2 & \\
\hline Median & 2 & & 1 & \\
\hline 0 & 0 & $(0.0)$ & 11 & (6.67) \\
\hline 1 & 8 & (4.8) & 152 & $(92.12)$ \\
\hline 2 & 105 & $(63.6)$ & 2 & (1.21) \\
\hline 3 & 34 & (20.6) & & \\
\hline 4 & 12 & $(7.3)$ & & \\
\hline 5 & 3 & (1.8) & & \\
\hline 6 & 1 & $(0.6)$ & & \\
\hline 7 & 2 & (1.2) & & \\
\hline
\end{tabular}

\section{Events at insertion}

There were no insertion failures, and neither perforations nor PID cases were reported during, or immediately following, insertion.

\section{Life table rates}

Table 3 gives the gross cumulative termination rates. The longest follow-up was 53.57 months and the shortest 1.11 months. There were three removals for abnormal bleeding at 1, 5 and 13 months after insertion. Another removal was done at 2.5 months which was not related to IUD use ('other reasons'). All other women continued to use the method
Table 3 Gross cumulative event rates per 100 GyneFix users

\begin{tabular}{lcc}
\hline Events & $\mathrm{n}$ & $\%$ \\
\hline Complete expulsion & 0 & 0.0 \\
Partial expulsion & 0 & 0.0 \\
Pregnancy & 0 & 0.0 \\
Perforation & 0 & 0.0 \\
Pain & 0 & 0.0 \\
Bleeding & 3 & 1.71 \\
Pain and bleeding & 0 & 0.0 \\
Pelvic inflamm. dis. (PID) & 0 & 0.0 \\
Other medical reasons & 0 & 0.0 \\
Pregnancy wish & 0 & 0.0 \\
Other personal reasons & 0 & 0.0 \\
Lost to follow-up & 0 & 0.0 \\
Other non-medical reasons & 1 & 0.57 \\
Total no. of terminations & 4 & 2.29 \\
Continuation of use & 171 & 97.71 \\
Total women-months & 1616 & \\
Min. women-months & 1.11 & \\
Max. women-months & 53.57 & \\
No. of women & 175 & \\
Women-Years & 134.6 & \\
\hline
\end{tabular}

(continuation rate 97.71). During the observation period, covering a total of 1616 women-months, no pregnancies or expulsions were reported. The follow-up in this study was $100 \%$.

\section{Discussion}

This is the second report of a study with the GyneFix IUD conducted in China. The first study reported on a 3-year, randomised, comparative, 'interval' study comparing the GyneFix IUD with the TCu380A IUD in parous women. ${ }^{2}$ This study showed a significantly lower use-related discontinuation rate at 3 years with the GyneFix IUD (8.34) than with the TCu380A IUD (14.13), and a higher rate of continuation with the GyneFix IUD compared to the TCu380A IUD (90.73 vs 85.25). Neither perforations nor PID cases were encountered with either device in this study, demonstrating the safety of the anchoring system. No pregnancies were reported with the frameless IUD. The follow-up in this study was almost $100 \%$ and the total women-months of experience approximately 10000.

When selecting an IUD for immediate post-abortal insertion, the performance characteristics should be taken into account. The IUD should be highly effective with a low expulsion rate, and should be well tolerated. Many women undergoing termination of pregnancy are nulliparous, or are women with a small uterus. This is probably more the case in China because of the population policy of allowing only one child per family.

In China, there are over 200 million contraceptive users (prevalence of use $>80 \%$ ). Among them, over 80 million use IUDs. A $2 \%$ failure rate per women-year means more than 1 million unwanted pregnancies. A 5\% incidence of side-effects means 4 million users suffering from bleeding, pain, or other adverse effects. Therefore, it is necessary to improve technology and develop new IUDs. ${ }^{4}$

The first study on the use of the frameless IUD in postabortal women reported on 125 GyneFix insertions in women with pregnancies of less than 10 weeks gestation (approximately 1000 women-months of experience), of which a large proportion were inserted in nulliparous women. Neither pregnancies nor expulsions were recorded and there were no complications (i.e. PID, perforation). ${ }^{17} \mathrm{~A}$ second report was made by Gbolade on the initial UK experience in 44 post-abortal women with similar results of high performance and absence of complications. ${ }^{18}$ 
Insertions in the latter study were performed in women up to 13 weeks amenorrhea immediately after interruption of the pregnancy on condition that the uterus was firmly contracted.

The majority of women in the present study experienced a previous induced abortion. Highly effective, well tolerated methods, particularly those that do not depend on daily administration, could be helpful in reducing the number of unplanned pregnancies and induced abortions. The frameless IUD is an effective and well tolerated IUD. The present study confirms previous studies conducted with the GyneFix IUD when inserted immediately following pregnancy termination of less than 10 weeks amenorrhea. As long as the provider has become experienced with this new technique of insertion, this IUD could be a welcome new contraceptive option.

Further data are being collected in a current multicentre clinical trial, initiated in 1998 in 10 centers in China, in which our centre is participating. The 6-month results in 212 women (1 254 women-months of experience) support the present results of high performance and absence of complications. ${ }^{19}$ These data will be reported at a later date.

\section{Conclusion}

Immediate post-abortal insertion of the frameless GyneFix IUD is an effective contraceptive option that could have significant world-wide impact on reducing the rate of repeat abortions.

Aknowledgement

The authors thank Dr D Wildemeersch for providing the GyneFix IUDs.
Statements on funding and competing interests

Funding.The GyneFix have been provided free of charge.

Competing interests. Dr D Wildemeersch is Clinical Research Director of Contrel Europe.

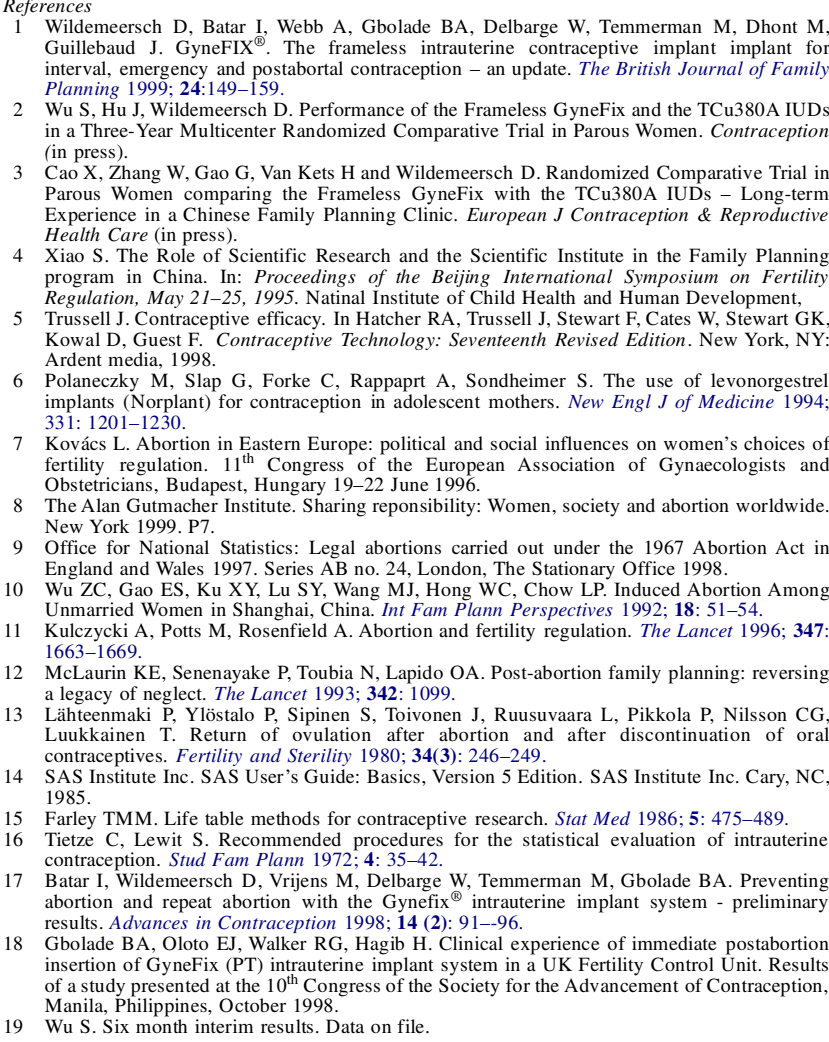

Wildemeersch D, Batar I, Webb A, Gbolade BA, Delbarge W, Temmerman M, Dhont M, Guillebaud J. GyneFIX ${ }^{\infty}$. The frameless intrauterine contraceptive implant implant for interval, emergency and postabortal contraception - an update. The British Journal of Family

Wu S, Hu J, Wildemeersch D. Performance of the Frameless GyneFix and the TCu380A IUDs in a Three-Year Multicenter Randomized Comparative Trial in Parous Women. Contraception (in press).

Cao X, Zhang W, Gao G, Van Kets H and Wildemeersch D. Randomized Comparative Trial in

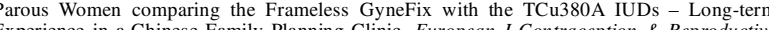
Health Care (in press) Xiao $\mathrm{S}$. The Role of

Xiao S. The Role of Scientific Research and the Scientific Institute in the Family Planning program in China. In: Proceedings of the Beijing International Symposium on Fertility

Regulation, May 21-25, 1995. Natinal Institute of Child Health and Human Development, Kowal D, Guest F. Contraceptive Technology: Seventeenth Revised Edition. New York, NY: Ardent media, 1998

6 Polaneczky M, Slap G, Forke C, Rappaprt A, Sondheimer S. The use of levonorgestrel implants (Norplant) for contraception in adolescent mothers. New Engl J of Medicine 1994

Kovács L. Abortion in Eastern Europe: political and social influences on women's choices of fertility regulation. $11^{\text {th }}$ Congress of the European Association of Gynaecologists an

Wociety and abortion worldwide. Office for National

(the 1967 Abortion Act in

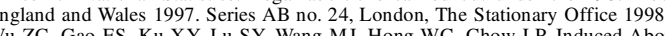

Wu ZC, Gao ES, Ku XY, Lu SY, Wang MJ, Hong WC, Chow LP. Induced Abortion Among Kulczycki A, Potts M, Rosenfield A. Abortion and fertility regulation. The Lancet 1996; 347: $1663-1669$.

McLaurin KE, Senenayake P, Toubia N, Lapido OA. Post-abortion family planning: reversin a legacy of neglect. The Lancet 1993; 342: 1099 .

Lahteenmaki P, Ylöstalo P, Sipinen S, Toivonen J, Ruusuvaara L, Pikkola P, Nilsson CG, Luukkainen T. Return of ovulation after abortion and after discontinuation of ora contraceptives. Fertility and Sterility 1980; 34(3): 246-249.

Version 5 Edition. SAS Institute Inc. Cary, NC,

15. Farley TMM. Life table methods for contraceptive research. Stat Med 1986; 5: 475-489.

Tietze C, Lewit S. Recommended procedures for the statistical evaluation of intrauterine contraception. Stud Fam Plann 1972; 4: 35-42.

W. Temmerman M, Gbolade BA. Preventing

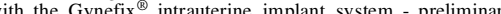
results. Advances in Contraception 1998; 14 (2): 91--96.

18 Gbolade BA, Oloto EJ, Walker RG, Hagib H. Clinical experience of immediate postabortion insertion of GyneFix (PT) intrauterine implant system in a UK Fertility Control Unit. Results of a study presented at the $10^{\text {th }}$ Congress of the Society for the Advancement of Contraception, Manila, Philippines, October 1998

19 Wu S. Six month interim results. Data on file.

\section{IMPORTANT NOTICE}

Since the introduction of the frameless GyneFix IUD in the UK, there has been approximately one uterine perforation per 1000 insertions. This rate is higher than the perforation rate reported during extensive international clinical trials in over 6000 women (no perforations were reported in these trials), and is similar to the perforation rate observed with conventional IUDs. ${ }^{1}$ Contrel UK, who market the GyneFix in the UK, in collaboration with Family Planning Sales, would like to make the following recommendations in order to minimise the risk of perforation:

- Always conduct a pelvic examination to establish the position of the uterus.

- Ensure alignment of the cervical canal and uterine cavity as the inserter is not very flexible.

- Always sound the uterus to know the direction and depth of the cavity.

- Do not proceed with the insertion if sounding is impossible or if you have any doubt.

- Insert the GyneFix applicator until you reach the fundus and keep it in contact with the fundus until you release the thread.

- Concentrate whilst placing the anchoring knot slowly and gently (mm by $\mathbf{m m}$ ) into the fundal wall.

- Check with an ultrasound scan if you have any doubt and remove the implant if it is not found to be in the correct position.

Doctors have reported that some GyneFix trainers instruct trainees to accomplish GyneFix anchoring by exerting one firm forward movement. This is not the correct procedure.

When you follow the recommendations set out above, the risk of perforation will be greatly reduced.

Please, let us know if you have any doubt about your insertion technique or need assistance (Phone 01981250682 and ask for Tim or Alison Anscomb).

\section{Dr D Wildemeersch}

Gynaecologist, Contrel Research, Belgium

Reference

Wildemeersch D, Batar I, Webb A, Gbolade BA, Delbarge W, Temmerman M, Dhont M, Guillebaud J. GyneFIX ${ }^{\circledR}$. The frameless intrauterine contraceptive implant implant for interval, emergency and postabortal contraception - an update. The British Journal of Family Planning 1999; 24: 149-159. 